\title{
Parameter Recognition of Mode-Converted Wave in Single-Source Ultrasound Using Gabor Transform for Bolt Axial Stress Evaluation
}

\author{
Ping Chen $\mathbb{D}^{1},{ }^{1}$ Xingliang He, ${ }^{1}$ and Wei Song ${ }^{2}$ \\ ${ }^{1}$ College of Mechanical Engineering, Chongqing University, Chongqing 400044, China \\ ${ }^{2}$ Southwest Oil and Gas Field Company, Chongqing 400021, China \\ Correspondence should be addressed to Ping Chen; chempion@126.com
}

Received 17 June 2020; Revised 21 July 2020; Accepted 5 August 2020; Published 20 August 2020

Academic Editor: Bin Gao

Copyright (c) 2020 Ping Chen et al. This is an open access article distributed under the Creative Commons Attribution License, which permits unrestricted use, distribution, and reproduction in any medium, provided the original work is properly cited.

\begin{abstract}
In this paper, a novel parameter recognition method of mode-converted wave in single-source ultrasound is put forward and applied to the estimation of axial stresses in bolts. To overcome the distortion and aliasing of the mode-converted wave in single-source ultrasonic signal, a time-frequency parameter recognition method based on the Gabor transform is introduced to recognize the accurate time of flight (TOF) of the mode-converted wave. Based on the mode conversion of the single-source longitudinal ultrasound, a new nonlinear evaluation model based on acoustoelastic equation is derived to determine the axial stress of bolt. The performance of the proposed method is evaluated by comparing it with the commonly used TOF ratio method of longitudinal wave and shear wave (L-S). The experiment result shows that the proposed method is more effective in detecting the connection status of bolted joints than the traditional L-S method.
\end{abstract}

\section{Introduction}

Loosening is one kind of failure that can markedly impact the stiffness and reliability of bolted joints. Insufficient preload of bolt will significantly increase the risk of overall mechanical structural disintegration. One way to address the critical issues in maintaining the strength of the structure and ensuring the safety of engineers is to apply appropriate pretightening force to the bolted joints and conduct long-term online monitoring of their axial stress. The most frequently used nondestructive estimation methods of stress include the photoelasticity method [1], resistance strain gauge method [2], piezoresistance method [3], active sensing method [4, 5], and ultrasonic testing method [6-9]. Among all these methods, the ultrasonic testing method is considered as a potential alternative technique due to its convenience to measure bolt tension with better accuracy.

Among the ultrasonic testing technologies, the acoustoelastic method by using TOF [6-12] is a typical stress measurement method. In the method, the TOF difference before and after bolt loading is calculated and multiplied with an acoustic-stress factor to achieve the axial stress. Whereas one shortcoming of this method is that it cannot be utilized to measure the stress of a fastened bolt. Yasui et al. [13] proposed the L-S method based on multisource ultrasound to solve this problem, in which the stress is measured according to the TOF ratio of longitudinal wave (L-wave) and shear wave (S-wave) by two separate transducers mounted on the two ends of the bolt with great care and precision. And the TOF ratio was proved to be independent of the original length of the bolt or the initial TOF. However, the coupling conditions (e.g., installation position, pressure, and coupling agent concentration) of the L-wave and S-wave transducers must be repeatable to ensure accuracy. Hence, this method is difficult to achieve reliable measurement results under practical conditions. Kim and Hong [14] proposed a stress measurement method using mode conversion of longitudinal waves to reduce the experimental error caused by different contact condition. However, compared with the multi-source method, owing to aliasing and distortion in the mode-converted wave signal used in Kim's method, it's difficult to obtain accurate TOF directly. They used a special designed probe with an acoustic lens to 
generate the mode-converted wave with high SNR (signalnoise ratio). In addition, it is necessary to provide enough space to install the specially designed probe, and the length of the workpiece cannot exceed the focal length of the lens. Therefore, a new parameter recognition method of the mode-converted wave in single-source ultrasound must be studied to promote the measurement efficiency of the bolt.

In this paper, a novel axial stress evaluation method using the mode-converted wave generated by single-source ultrasound based on the Gabor transform is proposed. Firstly, the distortion and aliasing of mode-converted process in single-source ultrasonic longitudinal signal are analyzed. Then, the parameter recognition method based on the Gabor transform and Gaussian empirical model to obtain the accurate TOF of the mode-converted signal is introduced and its effectiveness is proved by simulation. Next, a new nonlinear model for evaluating the axial stress of bolt is derived based on the acoustoelastic equation. Finally, the performance of the proposed method is evaluated by comparing it with the commonly used TOF ratio method of longitudinal wave and shear wave. And the experiment result shows that the proposed method is more effective in detecting the connection status of bolted joints than the traditional L-S method.

\section{TOF Recognition Method in Single- Source Ultrasound}

2.1. Mode-Converted Wave in Ultrasonic Testing. The velocities and polarization directions of the S-wave and the L-wave are related to the elastic properties, anisotropy, and current stress state of materials. Both the L-wave and the S-wave can be transmitted in solid medium, while only the L-wave can be transmitted in fluid medium. When a longitudinal wave beam enters the solid medium from the fluid medium, it will not only be reflected at the interface but will also derive an orthogonal bipolarized transverse wave and some other waveforms near the interface, such as the Rayleigh wave and the Love wave. Similarly, when there exist ultrasound incidents from the solid medium to the fluid medium, reflection wave and orthogonal bipolarized transverse wave will occur. This phenomenon is called mode conversion. Figure 1 shows a typical mode conversion process in a monostatic pulseecho experiment, in which the red beam represents a longitudinal wave and the green beam represents a shear wave.

In Figure 1, $\mathrm{L}_{\text {inc }}$ represents the incident single-source longitudinal wave generated by the transducer. At the liquidsolid interface, $\mathrm{L}_{\text {inc }}$ derives a reflection wave $\mathrm{L}_{\text {reff }}$, a transmission L-wave (LL), and a transmission shear wave (LT). Suppose that the reflection angle of the longitudinal wave is $\alpha$ and the angle of shear wave is $\beta$, the relationship between them can be described by Snell's law:

$$
\frac{\sin \alpha}{C_{L}}=\frac{\sin \beta}{C_{T}} .
$$

In equation (1), $C_{L}$ and $C_{T}$ refer to the acoustical velocities of $\mathrm{L}$-wave and $\mathrm{S}$-wave in the solid medium, respectively. When LL and LT reach the other end of the mounted solid, the mode conversion occurs again. The LL generates the LLL wave and

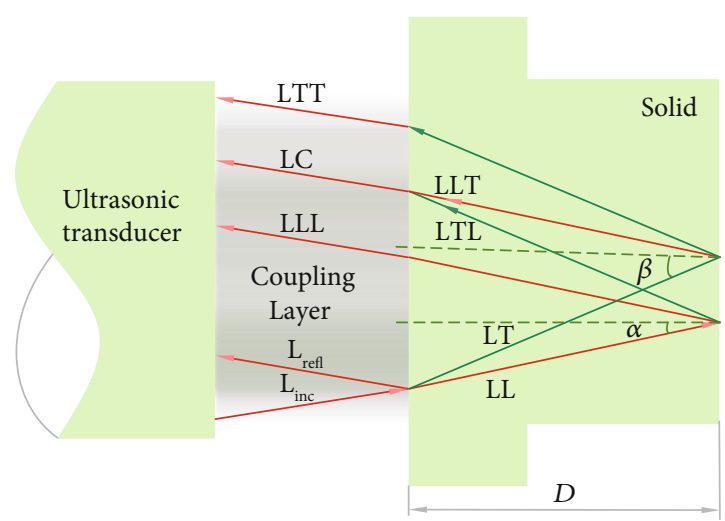

Figure 1: Mode conversion in a pulse-echo experiment.

the LLT wave, and the LT generates the LTL wave and the LTT wave. At last, all the waves undergo mode conversion again after arriving at the left solid interface, and the part transmitted into the coupling layer will all be converted to longitudinal waves and captured by the transducer. It should be noted that the propagation distance of the LLT and the LTL is exactly the same theoretically.

The transit time of acoustical wave can be rephrased as TOF, and $\mathrm{TOF}_{L}$ and $\mathrm{TOF}_{T}$ denote the transit time of $\mathrm{L}$ wave and $\mathrm{S}$-wave, respectively, in this paper. The relationship between the TOF and the sound velocity can be expressed as

$$
\left\{\begin{array}{l}
C_{L} \approx \frac{2 D}{\mathrm{TOF}_{L}}, \\
C_{T} \approx \frac{2 D}{\mathrm{TOF}_{T}},
\end{array}\right.
$$

where $D$ is the length of the solid medium. And the TOF of the converted waves $\left(T_{\text {LLL }}, T_{\text {LLT }}, T_{\text {LTL }}\right.$, and $\left.T_{\text {LTT }}\right)$ in solid can be expressed as

$$
\left\{\begin{array}{l}
T_{\mathrm{LLL}}=\frac{2 D / \cos \alpha}{2 D / \mathrm{TOF}_{L}}, \\
T_{\mathrm{LLT}}=\frac{D / \cos \alpha}{2 D / \mathrm{TOF}_{L}}+\frac{D / \cos \beta}{2 D / \mathrm{TOF}_{T}}, \\
T_{\mathrm{LTL}}=\frac{D / \cos \alpha}{2 D / \mathrm{TOF}_{L}}+\frac{D / \cos \beta}{2 D / \mathrm{TOF}_{T}}, \\
T_{\mathrm{LTT}}=\frac{2 D / \cos \beta}{2 D / \mathrm{TOF}_{T}} .
\end{array}\right.
$$

When the incident direction is vertical to the interface, the angles $\alpha$ and $\beta$ are approximately equal to zero. So, their relationship can be described as

$$
T_{\text {LLL }}<T_{\text {LLT }}=T_{\text {LTL }}<T_{\text {LTT }} .
$$

Equations (3) and (4) can determine the positions of the converted waves in a time-domain signal approximately. In general, the LTT wave has serious attenuation in amplitude and falls in the vicinity of the second echo of LLL (because 
the travel speed of the L-wave is nearly two times than that of the S-wave). Therefore, the LTT wave is not suitable for stress evaluation. Owing to the relatively low attenuation, it is possible for the remaining LLT wave and the LTL wave to be adopted in stress evaluation. However, their waveforms are completely overlapped in the time domain because the LLT and LTL have exactly the same TOF in theory (here we use LC to represent the overlapped mode-converted waveforms). Moreover, LC often exhibits varying degrees of nonlinear distortion and aliasing caused by coupling conditions and multiple mode conversion. As a result, the direct acquisition of the LC TOF through the original signal is almost infeasible.

2.2. Parameter Recognition Method Using the Gabor Transform. The cross-correlation method is the most commonly used parameter recognition method to obtain the TOF of an ultrasonic signal, which can achieve high estimation accuracy under the condition of high signal-to-noise ratio (SNR). However, due to the nonlinear distortion and aliasing in LC, applying the cross-correlation method to this situation may cause extra error [15]. This paper introduces an ultrasonic signal processing method based on an empirical model $[15,16]$ and the Gabor transform [17] to recognize the parameter of the LC. The Gabor transform is a timefrequency transform method and widely used in signal parameter estimation for the ability of revealing the local frequency distribution of a signal or an image. From the perspective of time-frequency analysis, this paper applies the Gabor transform to estimate the parameter of the empirical model of LC. The authors use $X_{\mathrm{LC}}(t)$ to express the signal of LC in time domain, and $X_{\mathrm{LC}}(t)$ can be rephrased as a two-dimensional time-frequency expansion by applying the Gabor transform:

$X_{\mathrm{LC}}(t)=\sum_{\tau=-\infty}^{+\infty} \sum_{\omega=-\infty}^{+\infty} C_{\tau, \omega} h_{\tau, \omega}=\sum_{\tau=-\infty}^{+\infty} \sum_{\omega=-\infty}^{+\infty} C_{\tau, \omega} h(t-\tau a) e^{-j 2 \pi b \omega t}$.

In equation (5), $\tau$ and $\omega$ represent the time and frequency coordinates of the two-dimensional plane, $a$ and $b$ are the width of the time and frequency grid of the plane, respectively, $C_{\tau, \omega}$ is the Gabor expansion coefficient, and $h_{\tau, \omega}$ is the Gabor generating function. What is more, $C_{\tau, \omega}$ is also defined as the Gabor transform (GT) of $X_{\mathrm{LC}}(t)$ :

$$
C_{\tau, \omega}=\mathrm{GT}\left(X_{\mathrm{LC}}\right)=\int_{-\infty}^{\infty} X_{\mathrm{LC}}(\delta) h^{*}(\delta-\tau a) e^{-j 2 \pi \omega b \delta} d \delta
$$

According to the physical characteristics of the ultrasonic transducer commonly used in bolt stress detection technology, the Gauss attenuation signal [18] is selected as the empirical model to describe LC:

$$
S_{\mathrm{LC}}(t)=A_{0} e^{(-d(t-\tau))^{2}} \sin 2 \pi \omega(t-\tau) .
$$

In equation (7), $A_{0}$ is the amplitude and $d$ is the shape factor. Since the Gaussian function is still a Gaussian kernel function after Fourier transformation, its energy distribution is concentrated in both the frequency domain and the time domain, which can clearly reveal the local details of the signal. Considering that $S_{\mathrm{LC}}(t)$ is a Gaussian model, an analysis window function with a Gaussian kernel is selected as the Gabor generating function:

$$
h_{\tau, \omega}=\eta e^{-r_{0}(t-a \tau)^{2}+j \omega_{0}[(t-a \tau) / b \omega]} .
$$

To ensure the energy uniformity of the window function, let

$$
\eta=\left(\frac{2 r_{0}}{\pi}\right)^{1 / 4}
$$

Then, the Euclidean distance of Gabor generating function and the empirical model is adopted to characterize the similarity between $S_{\mathrm{LC}}(t)$ and $h_{\tau, \omega}(t)$, which can be expressed as

$$
\left\|S_{\mathrm{LC}}(t)-h_{\tau, \omega}(t)\right\|^{2}=S_{\mathrm{LC}}{ }^{2}(t)+h_{\tau, \omega}{ }^{2}(t)-2\left|\mathrm{GT}\left(S_{\mathrm{LC}}\right)\right|
$$

In fact, the purpose of finding the maximum similarity between the Gabor generating function and the empirical model is equivalent to obtaining the minimum value of equation (10). Since $S_{\mathrm{LC}}{ }^{2}(t)$ and $h_{\tau, \omega}^{2}(t)$ are both nonnegative and unrelated to $\tau$ and $\omega$, the minimum value of equation (10) is transformed into the maximum value of $\left|\mathrm{GT}\left(S_{\mathrm{LC}}\right)\right|$. And $\mid \mathrm{GT}$ $\left(S_{\mathrm{LC}}\right) \mid$ can be further written as

$$
\left|\mathrm{GT}\left(S_{\mathrm{LC}}\right)\right|=f(\tau, \omega)=\eta A_{0} \sqrt{\frac{\pi}{d+r_{0}}} e^{-\left(\left(4 d r_{0}(\tau-\mathrm{TOF})^{2}+\left(\omega_{c}-\left(\omega_{0} / \omega\right)\right)^{2}\right) /\left(4\left(d+r_{0}\right)\right)\right)} .
$$

In conclusion, the parameter recognition of the empirical model of LC can be described as

$$
\left\{\mathrm{TOF}, \omega_{C}\right\}=\arg \max _{\tau, \omega}\left|\mathrm{GT}\left(S_{\mathrm{LC}}\right)\right|
$$

2.3. Parameter Recognition Using Particle Filter. Absolutely, the solution of equation (12) is a typical two-dimensional optimization problem. Taking both estimation accuracy and convergence speed into account, the particle filter method is selected as the solver of equation (12). The basic idea of the particle filter algorithm is the Monte Carlo method, which approximates and replaces the probability density function of the system through a large number of random samples and selects a subset of particles for sequential importance sampling according to the posterior probability in order to shrink the scope of estimation [19-22]. Assume the target vector $V_{\mathrm{LC}}$ :

$$
V_{\mathrm{LC}}=\left(\mathrm{TOF}, \omega_{\mathrm{C}}\right)
$$

The parameter recognition process of the empirical model of LC based on the particle filter method is as follows:

(1) Suppose the lengths of the time and the frequency axials of the Gabor expansion plane is $M$ and $N$, respectively. Establish the initial particle set according to the number of particles $K$ : 


$$
\begin{gathered}
P\left({ }^{0} V_{\mathrm{LC}}\right)=\left\{{ }^{0} V_{\mathrm{LC}}{ }^{i}, W_{0}{ }^{i}\right\}_{i=0}^{K}, \\
\sum_{i=0}^{N} W_{0}{ }^{i}=1 .
\end{gathered}
$$

In equation (14), ${ }^{0} V_{\mathrm{LC}}{ }^{i}$ refers to the target vector of each particle in the initial set and $W_{0}$ is the probability of each particle in the initial set. They can be expressed as

$$
\left\{\begin{array}{l}
{ }^{0} V_{\mathrm{LC}^{i}}=\left(\mathrm{TOF}_{0}{ }^{i}, \omega_{C 0}{ }^{i}\right), \\
\mathrm{TOF}_{0}{ }^{i} \in[0, M], \omega_{C 0}{ }^{i} \in[0, N] .
\end{array}\right.
$$

To ensure generality, the uniform distribution is selected as the initial importance distribution:

$$
W_{0}^{i}=\left(\frac{1}{M}, \frac{1}{N}\right)
$$

(2) Substituting the value of $\mathrm{TOF}_{0}{ }^{i}$ and $\omega_{\mathrm{C} 0}{ }^{i}$ into equation (11) for each particle in the set, then the expression of $\left|\mathrm{GT}_{i}\left(S_{\mathrm{LC}}\right)\right|$ can be derived. Next, we can calculate the state transition probability according to the likelihood between $\left|\mathrm{GT}\left(S_{\mathrm{LC}}\right)\right|$ and $\mid \mathrm{GT}_{i}\left(X_{\mathrm{LC}}\right)$ I. The normalized weight corresponding to the state transition probability of each particle is given by

$$
w_{i}=\frac{\left|\mathrm{GT}_{i}\left(S_{\mathrm{LC}}\right)\right|-\left|\mathrm{GT}\left(X_{\mathrm{LC}}\right)\right|}{\sum_{i=1}^{K}\left|\mathrm{GT}_{i}\left(S_{\mathrm{LC}}\right)\right|-\left|\mathrm{GT}\left(X_{\mathrm{LC}}\right)\right|} .
$$

(3) Perform sequential importance resample according to the elimination threshold which equals to the average of $w_{i}$. The particles with low weight are eliminated and those with high weight are retained and copied. After resample, the new particle set can be derived as

$$
\begin{gathered}
P\left({ }^{j} V_{\mathrm{LC}}\right)=\left\{{ }^{j} V_{\mathrm{LC}}{ }^{i}, W_{j}{ }^{i}\right\}_{i=0}^{K}, \\
\sum_{i=0}^{N} W_{j}^{i}=1 .
\end{gathered}
$$

(4) Repeat procedures (1) to (3) until the values of every particle are exactly the same as each other, and the target vector of the last set is expressed as

$$
V_{\mathrm{LC}}{ }^{\text {est }}=\left(\mathrm{TOF}^{\mathrm{est}}, \omega_{C}{ }^{\text {est }}\right)
$$

TABLe 1: Parameters of the simulation signals.

\begin{tabular}{lcccc}
\hline Number & $f_{c_{1}}(\mathrm{MHz})$ & $f_{c_{2}}(\mathrm{MHz})$ & $\tau_{1}(\mathrm{~s})$ & $\tau_{2}(\mathrm{~s})$ \\
\hline 1 & 4.000 & 3.000 & $1.900 \times 10^{-6}$ & $2.100 \times 10^{-6}$ \\
2 & 4.500 & 3.500 & $2.000 \times 10^{-6}$ & $2.200 \times 10^{-6}$ \\
3 & 5.002 & 4.000 & $2.100 \times 10^{-6}$ & $2.300 \times 10^{-6}$ \\
4 & 5.500 & 4.500 & $2.200 \times 10^{-6}$ & $2.400 \times 10^{-6}$ \\
5 & 6.000 & 5.000 & $2.300 \times 10^{-6}$ & $2.500 \times 10^{-6}$ \\
6 & 6.500 & 5.500 & $2.400 \times 10^{-6}$ & $2.600 \times 10^{-6}$ \\
7 & 7.000 & 6.000 & $2.500 \times 10^{-6}$ & $2.700 \times 10^{-6}$ \\
8 & 7.500 & 6.500 & $2.600 \times 10^{-6}$ & $2.800 \times 10^{-6}$ \\
\hline
\end{tabular}

TOF ${ }^{\text {est }}$ and $\omega_{C}{ }^{\text {est }}$ are corresponding to the TOF and the central frequency of mode-converted wave LC.

2.4. Simulation. To evaluate the performance of the proposed method, a group of double Gaussian echo signals with different TOF and central frequency are introduced to simulate the mode-converted wave LC. The general expression of the signals is

$S(t)=A_{1} e^{\left(-d_{1}\left(t-\tau_{1}\right)\right)^{2}} \sin f_{c_{1}}\left(t-\tau_{1}\right)+A_{2} e^{\left(-d_{2}\left(t-\tau_{2}\right)\right)^{2}} \sin f_{c_{2}}\left(t-\tau_{2}\right)$,

where $A_{1}$ and $A_{2}$ represent the amplitude of the echo envelope, $\tau_{1}$ and $\tau_{2}$ are the TOF, $f_{c_{1}}$ and $f_{c_{2}}$ are the central frequencies, and $d_{1}$ and $d_{2}$ are the shape factors. In each signal, $A_{1}=1 \mathrm{~V}, A_{2}=0.8 \mathrm{~V}, d_{1}=1.5 \times 10^{-7} \mathrm{~s}$, and $d_{2}=1.4 \times 10^{-7} \mathrm{~s}$. The values of the TOF and the central frequency of these signals are shown in Table 1. The initial set is made up of 10000 random chosen particles within the range of $\pm 2 \times 10-7 \mathrm{sec}$ and $\pm 0.5 \mathrm{MHz}$ around the preset values. After $-10 \mathrm{~dB} \sim 10 \mathrm{~dB}$ Gaussian white noise is applied to the signals to simulate different SNR conditions, the TOFs and the central frequencies of No. 1 No. 8 signals are recognized and each signal is calculated over 100 times. The No. 1 signal with $5 \mathrm{~dB}$ noise is shown in Figure 2(a), and its Gabor expansion is shown in Figure 2(b). It can be observed in Figure 2(a) that the timedomain signal is severely distorted and the echoes are submerged in clutter, while the echoes can be clearly recognized in the diagram of Gabor expansion (Figure 2(b)) and the recognized results are shown in Table 2.

The TOF difference caused by the acoustoelastic effect in an actual measurement is generally about $1 \%$, while for the proposed method the measurement error of TOF is about $0.5 \%$ in average under the condition of low SNR, as shown in Table 2. Therefore, the parameter recognition using the Gabor transform is efficient and can be applied to the TOF recognition of mode-converted wave in single-source ultrasound.

\section{Nonlinear Evaluation Model of Bolt Axial Stress}

3.1. Nonlinear Evaluation Model Based on Mode-Converted Ultrasound. The existing evaluation models [6-14] of bolt 


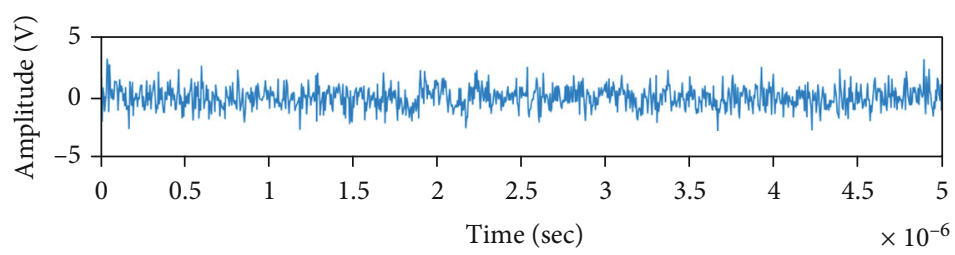

(a) Simulation signal

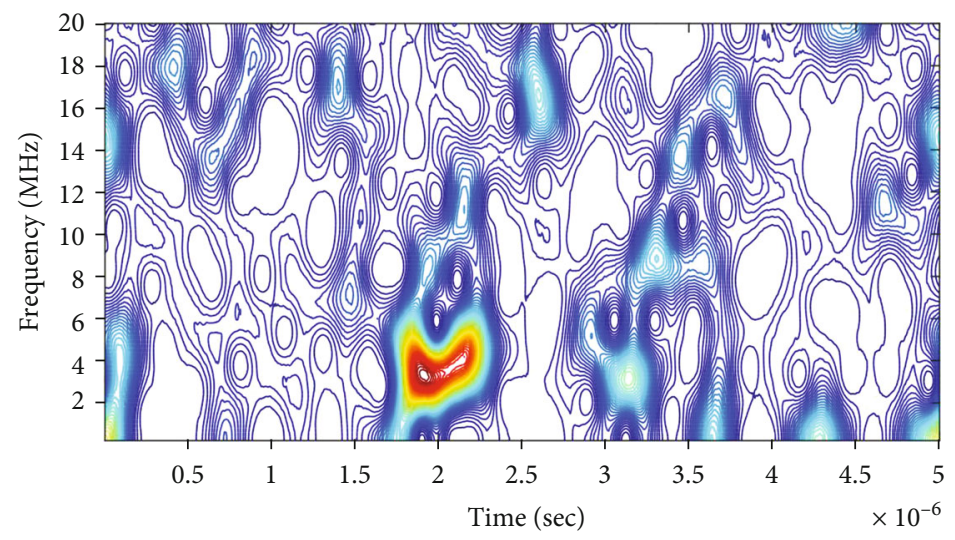

(b) Gabor expansion result

Figure 2: Simulation results.

TABLE 2: Recognized results of the simulation signals.

\begin{tabular}{lcccccc}
\hline Number & $f_{c_{1}}(\mathrm{MHz})$ & $f_{c_{2}}(\mathrm{MHz})$ & Average frequency error & $\tau_{1}(\mathrm{~s})$ & $\tau_{2}(\mathrm{~s})$ & Average TOF error \\
\hline 1 & 4.082 & 2.939 & $2.04 \%$ & $1.901 \times 10^{-6}$ & $2.106 \times 10^{-6}$ & $0.17 \%$ \\
2 & 4.495 & 3.507 & $0.15 \%$ & $1.987 \times 10^{-6}$ & $2.198 \times 10^{-6}$ & $0.76 \%$ \\
3 & 5.012 & 3.978 & $0.37 \%$ & $2.102 \times 10^{-6}$ & $2.308 \times 10^{-6}$ & $0.22 \%$ \\
4 & 5.498 & 4.487 & $0.24 \%$ & $2.204 \times 10^{-6}$ & $2.389 \times 10^{-6}$ & $0.32 \%$ \\
5 & 5.991 & 5.012 & $0.19 \%$ & $2.297 \times 10^{-6}$ & $2.486 \times 10^{-6}$ & $0.35 \%$ \\
6 & 6.504 & 5.612 & $0.89 \%$ & $2.406 \times 10^{-6}$ & $2.587 \times 10^{-6}$ & $0.38 \%$ \\
7 & 6.993 & 6.124 & $1.00 \%$ & $2.507 \times 10^{-6}$ & $2.712 \times 10^{-6}$ & $0.36 \%$ \\
8 & 7.522 & 6.514 & $0.25 \%$ & $2.602 \times 10^{-6}$ & $2.791 \times 10^{-6}$ & $0.20 \%$ \\
\hline
\end{tabular}

axial stress are simplified and linear. And it is inevitable for them to induce extra fitting error. In this part, a new nonlinear evaluation model of bolt axial stress is proposed, its nonlinear feature is much better to meet the characteristic of acoustic elastic effect in theory. According to the theory of elasticity and nonlinear acoustic [23],

$$
\left\{\begin{array}{l}
C_{L}{ }^{2}=C_{L 0}{ }^{2}-\sigma\left[\frac{(\lambda+2 \mu)(4 \lambda+10 \mu+4 m) /(\mu+\lambda+2 j)}{\rho_{0}(3 \lambda+2 \mu)}\right], \\
C_{T}{ }^{2}=C_{T 0}{ }^{2}-\sigma\left[\frac{(2 j-2(m+\lambda+2 \mu)) / \mu}{\rho_{0}(3 \lambda+2 \mu)}\right]
\end{array}\right.
$$

where $C_{L}$ and $C_{T}$ are the velocities of longitudinal wave and shear wave in a solid medium under stress, respectively; $C_{L 0}$ and $C_{T 0}$ are their velocities in the stress-free state; $\lambda$ and $\mu$ are the Lame coefficients; $j$ and $m$ are the third-order elastic constants and $\rho_{0}$ is the density of the solid. Equation (21) can be rewritten as

$$
\left\{\begin{array}{l}
C_{L}=C_{L 0}\left(1-k_{L} \sigma\right)^{1 / 2}, \\
C_{T}=C_{T 0}\left(1-k_{T} \sigma\right)^{1 / 2}
\end{array}\right.
$$

where

$$
\begin{gathered}
k_{L}=\frac{(\lambda+2 \mu)(4 \lambda+10 \mu+4 m) /(\mu+\lambda+2 j)}{\left[\rho_{0}(3 \lambda+2 \mu)\right]^{1 / 2} / C_{L 0}}, \\
k_{T}=\frac{(2 j-2(m+\lambda+2 \mu)) / \mu}{\left[\rho_{0}(3 \lambda+2 \mu)\right]^{1 / 2} / C_{T 0}} .
\end{gathered}
$$

The propagation speed of elastic waves will change along with the stress status of the medium on the basis of equation (22), while in practical measurement, because the change of 


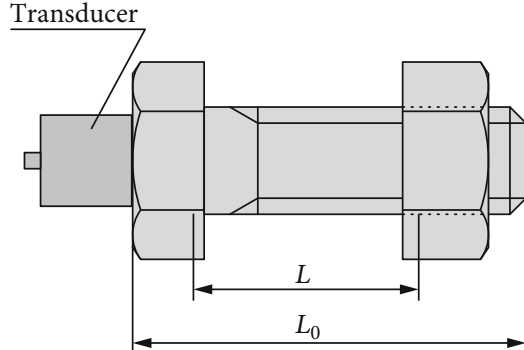

FIGURE 3: Bolt connection structure.

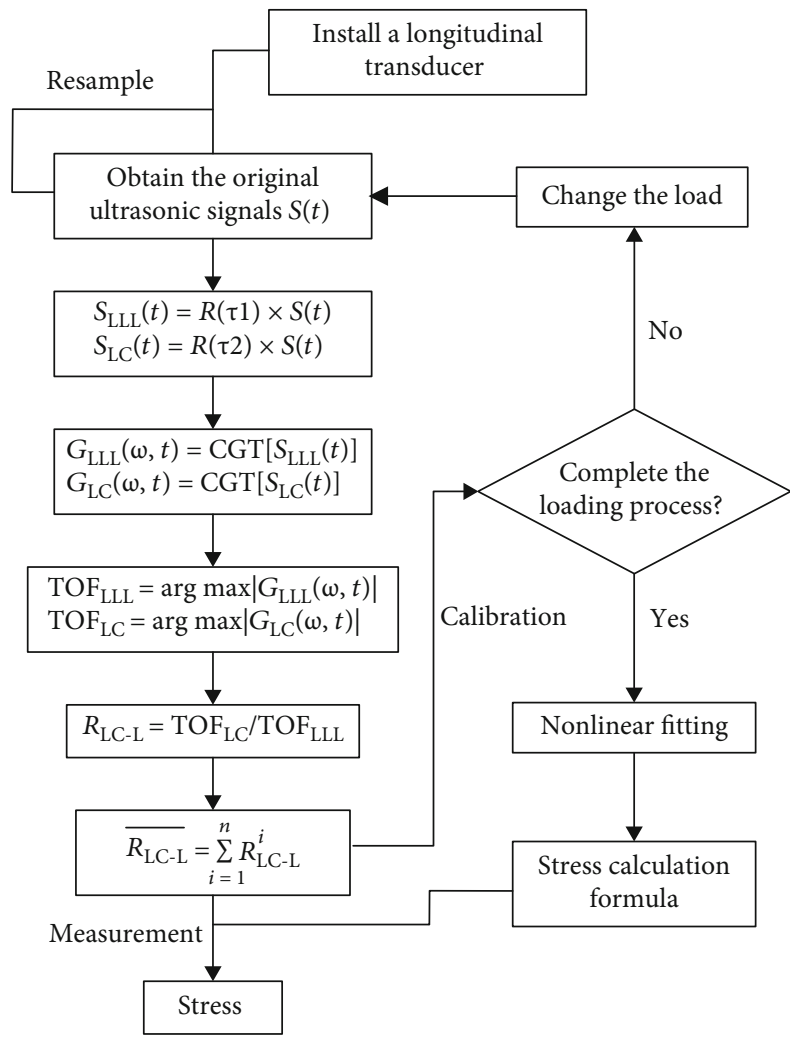

FIGURE 4: Flowchart of the stress evaluation.

sound velocity is too weak to detect, TOF is often frequently used instead of velocity. Figure 3 shows a typical bolt connection structure, in which $L$ and $L_{0}$ are the equivalent stress length and overall length of the bolt, respectively. Since the stress distribution in a loaded bolt is inhomogeneous, the bolt can be simplified to an axisymmetric cylinder with uniform stress distribution, in which $L$ represents the length of the cylinder. Then, the TOF can be expressed as

$$
\left\{\begin{array}{l}
T_{L}=\frac{2\left[\left(1+\sigma E^{-1}\right) L+L_{0}-L\right]}{C_{L}}, \\
T_{T}=\frac{2\left[\left(1+\sigma E^{-1}\right) L+L_{0}-L\right]}{C_{S}},
\end{array}\right.
$$

where $T_{L}$ and $T_{T}$ are the TOF of L-wave and S-wave, respectively. Squaring the quotient of $T_{L}$ by $T_{T}$, we can obtain

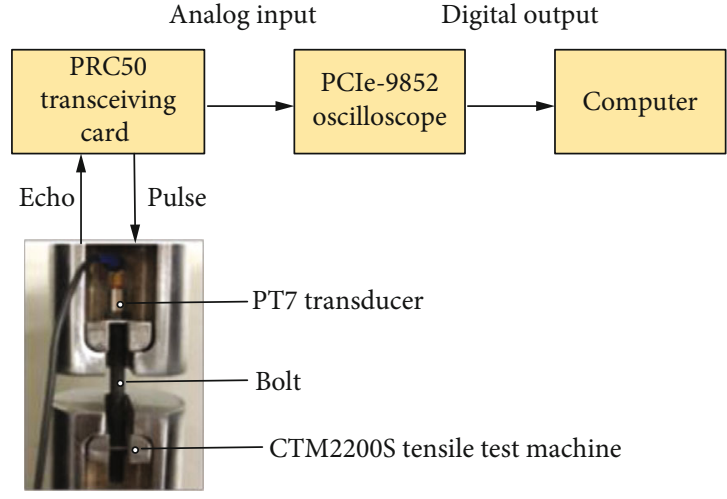

Figure 5: Experiment device.

$$
R_{\mathrm{L}-\mathrm{S}}{ }^{2}=\frac{C_{L 0}{ }^{2} K_{L}}{C_{T 0}{ }^{2} K_{T}} \frac{\left(C_{T 0}{ }^{2} K_{T} / C_{L 0}{ }^{2} K_{L}\right)-\left(C_{T 0}{ }^{2} / C_{L 0}{ }^{2}\right)}{\left(C_{T 0}{ }^{2} K_{T} / C_{L 0}{ }^{2} K_{L}\right)\left(\left(C_{T 0}{ }^{2} / C_{L 0}{ }^{2}\right)-\left(C_{T 0}{ }^{2} / C_{L 0}{ }^{2}\right) K_{T} \sigma\right)} .
$$

Let

$$
\begin{gathered}
a=\frac{C_{T 0}{ }^{2} K_{T}}{C_{L 0}{ }^{2} K_{L}}, \\
b=\frac{C_{T 0}^{2}}{C_{L 0}^{2}}, \\
c=K_{T} .
\end{gathered}
$$

Then, equation (25) can be simplified as

$$
R_{\mathrm{L}-\mathrm{S}}^{2}=\frac{1}{a}+\frac{a-b}{a(b-b c \sigma)} .
$$

Since the TOF of LC can be expressed as

$$
T_{\mathrm{LC}}=\frac{T_{T}+T_{L}}{2}=\frac{T_{L} / x+T}{2},
$$

the TOF ratio $R_{\mathrm{LC}-\mathrm{L}}$ of LC wave to LLL wave (LC-L method) is derived as

$$
R_{\mathrm{LC}-\mathrm{L}}=\frac{1}{2 R_{\mathrm{L}-\mathrm{S}}}+\frac{1}{2}
$$

Finally, the axial stress $\sigma$ can be calculated by the following nonlinear model:

$$
\sigma=\frac{b-a}{a b c R_{\mathrm{L}-\mathrm{S}}{ }^{2}-b c}+\frac{1}{c}=\frac{b-a}{a b c\left[1 /\left(2 R_{\mathrm{LC}-\mathrm{L}}-1\right)\right]^{2}-b c}+\frac{1}{c} .
$$

Based on the derivations above, as long as the coefficients related to material properties and the TOF ratio $R_{\mathrm{LC}-\mathrm{L}}$ are known, the axial stress of the bolt can be determined. 
TABLE 3: Details of the samples.

\begin{tabular}{lcccc}
\hline Type & Specification & Guaranteed load & Section area & Material \\
\hline \multirow{2}{*}{ Hexagon head half thread bolt } & M8 $\times 104$ & $2160 \mathrm{~kg}$ & $36.6 \mathrm{~mm}^{2}$ & C45 \\
& M12 $\times 200$ & $3200 \mathrm{~kg}$ & $84.3 \mathrm{~mm}^{2}$ & C35 \\
\hline
\end{tabular}

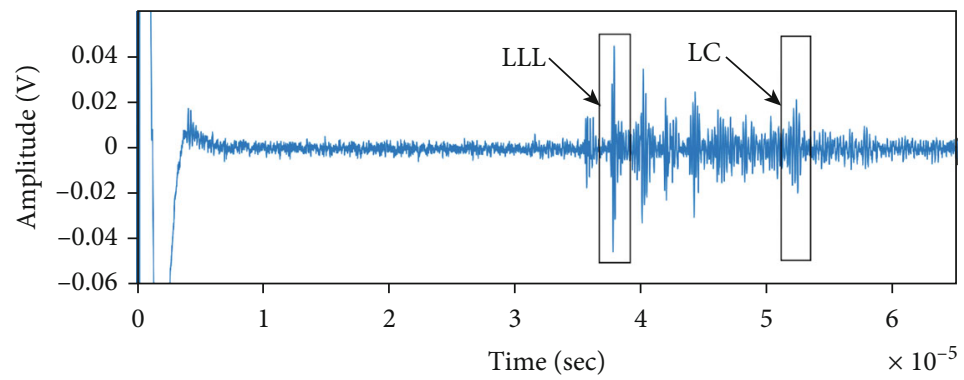

FIGURE 6: Ultrasound in a M8 × 104 bolt.

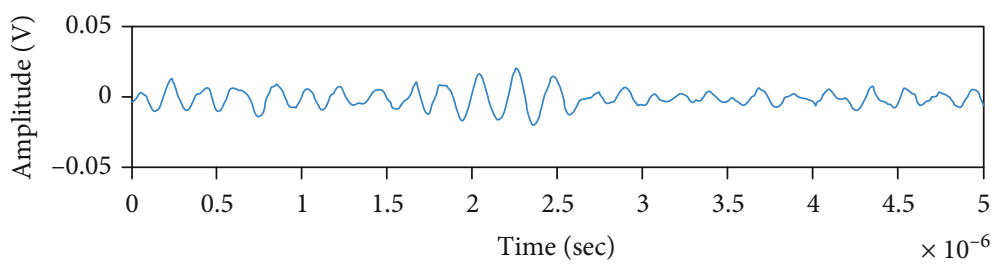

(a) LC waveform

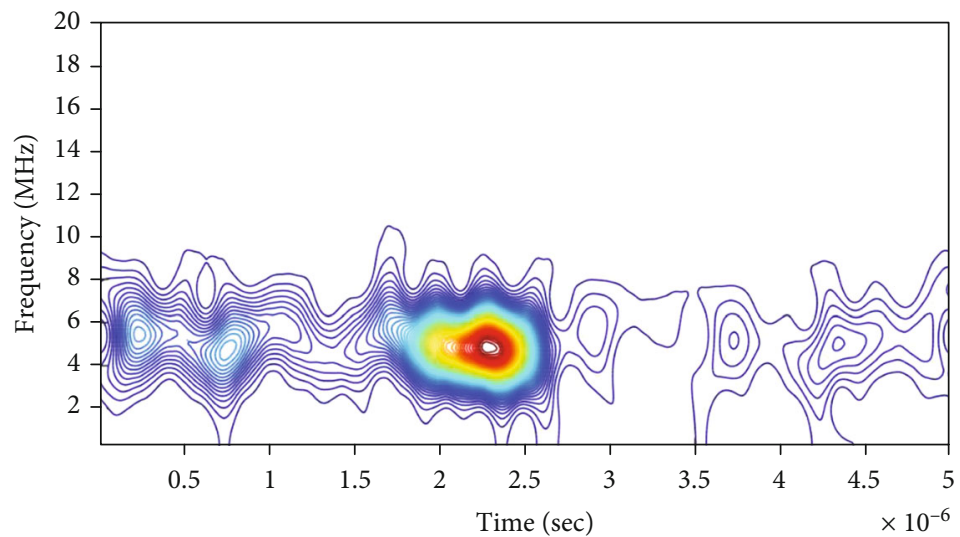

(b) Gabor expansion of LC

Figure 7: LC and its Gabor expansion.

3.2. Flowchart of Stress Evaluation. The process of the measurement and calibration methods of bolt axial stress using single-source mode-converted ultrasound is shown in Figure 4.

(1) Mill the head of the tested bolt flat and mount a longitudinal transducer on it properly to ensure stable coupling status

(2) Obtain the original signal by conducting a monostatic pulse-echo experiment, and then resample the signal for at least 100 times to get the statistical data of the signal
(3) Extract waveform of LLL $\left(S_{\mathrm{LLL}}(t)\right)$ and LC $\left(S_{\mathrm{LC}}(t)\right)$ of each signal by a rectangular window $R(\tau)$

(4) Utilize the Gabor transform to get the Gabor expansion of $S_{\mathrm{LLL}}(t)$ and $S_{\mathrm{LC}}(t)$

(5) Calculate the TOF of LC and LLL by the particle filter optimization method

(6) Obtain the value of $R_{\mathrm{LC}-\mathrm{L}}$ of each signal by dividing the TOF of LC to the TOF of LLL

(7) Average the statistical $R_{\mathrm{LC}-\mathrm{L}}$ and get the best estimate value to reduce the random errors 
TABLE 4: Estimated TOF of sample M8 × 104 .

\begin{tabular}{lccccc}
\hline $\begin{array}{l}\text { Axial stress } \\
(\mathrm{MPa})\end{array}$ & $\begin{array}{c}\text { TOF of L } \\
(\mu S)\end{array}$ & $\begin{array}{c}\text { TOF of } \\
\text { LC }(\mu S)\end{array}$ & $\begin{array}{c}\text { TOF of } \\
\text { S }(\mu S)\end{array}$ & $R_{\mathrm{LC}-\mathrm{L}}$ & $R_{\mathrm{L}-\mathrm{S}}$ \\
\hline 0 & 37.763 & 52.271 & 65.417 & 1.38419 & 0.57727 \\
10 & 37.770 & 52.279 & 65.427 & 1.38414 & 0.57728 \\
20 & 37.778 & 52.289 & 65.435 & 1.38411 & 0.57734 \\
30 & 37.785 & 52.298 & 65.449 & 1.38409 & 0.57732 \\
40 & 37.793 & 52.308 & 65.460 & 1.38407 & 0.57734 \\
50 & 37.799 & 52.316 & 65.471 & 1.38406 & 0.57734 \\
60 & 37.808 & 52.327 & 65.480 & 1.38402 & 0.57740 \\
70 & 37.815 & 52.336 & 65.491 & 1.38400 & 0.57741 \\
80 & 37.822 & 52.344 & 65.502 & 1.38396 & 0.57742 \\
90 & 37.829 & 52.353 & 65.513 & 1.38394 & 0.57743 \\
100 & 37.836 & 52.362 & 65.524 & 1.38392 & 0.57744 \\
110 & 37.842 & 52.370 & 65.534 & 1.38391 & 0.57744 \\
120 & 37.849 & 52.379 & 65.545 & 1.38389 & 0.57745 \\
130 & 37.856 & 52.387 & 65.556 & 1.38385 & 0.57746 \\
140 & 37.866 & 52.399 & 65.566 & 1.38380 & 0.57752 \\
150 & 37.874 & 52.408 & 65.577 & 1.38375 & 0.57755 \\
160 & 37.881 & 52.416 & 65.587 & 1.38370 & 0.57757 \\
170 & 37.887 & 52.424 & 65.598 & 1.38369 & 0.57756 \\
180 & 37.893 & 52.431 & 65.603 & 1.38366 & 0.57761 \\
190 & 37.901 & 52.439 & 65.621 & 1.38358 & 0.57757 \\
\hline & & & & &
\end{tabular}

TABLE 5: Estimated TOF of sample M12 200 .

\begin{tabular}{lccccc}
\hline $\begin{array}{l}\text { Axial stress } \\
(\mathrm{MPa})\end{array}$ & $\begin{array}{c}\text { TOF of L } \\
(\mu S)\end{array}$ & $\begin{array}{c}\text { TOF of } \\
\text { LC }(\mu S)\end{array}$ & $\begin{array}{c}\text { TOF of } \\
\text { S }(\mu S)\end{array}$ & $R_{\text {LC-L }}$ & $R_{\mathrm{L}-\mathrm{S}}$ \\
\hline 0 & 76.194 & 105.170 & 133.346 & 1.38029 & 0.57140 \\
10 & 76.207 & 105.186 & 133.365 & 1.38027 & 0.57142 \\
20 & 76.222 & 105.204 & 133.386 & 1.38023 & 0.57144 \\
30 & 76.236 & 105.221 & 133.415 & 1.38020 & 0.57142 \\
40 & 76.250 & 105.238 & 133.428 & 1.38017 & 0.57147 \\
50 & 76.265 & 105.256 & 133.450 & 1.38014 & 0.57149 \\
60 & 76.279 & 105.274 & 133.475 & 1.38012 & 0.57149 \\
70 & 76.292 & 105.289 & 133.489 & 1.38008 & 0.57152 \\
80 & 76.305 & 105.305 & 133.510 & 1.38005 & 0.57153 \\
90 & 76.315 & 105.317 & 133.530 & 1.38003 & 0.57152 \\
100 & 76.328 & 105.333 & 133.545 & 1.38000 & 0.57155 \\
110 & 76.343 & 105.351 & 133.573 & 1.37997 & 0.57155 \\
120 & 76.358 & 105.368 & 133.591 & 1.37992 & 0.57158 \\
130 & 76.373 & 105.386 & 133.621 & 1.37989 & 0.57156 \\
140 & 76.387 & 105.403 & 133.639 & 1.37986 & 0.57159 \\
150 & 76.402 & 105.421 & 133.658 & 1.37982 & 0.57162 \\
160 & 76.416 & 105.438 & 133.685 & 1.37979 & 0.57161 \\
170 & 76.430 & 105.456 & 133.701 & 1.37977 & 0.57165 \\
180 & 76.443 & 105.471 & 133.721 & 1.37973 & 0.57166 \\
190 & 76.452 & 105.481 & 133.738 & 1.37970 & 0.57166 \\
\hline & & & & &
\end{tabular}

(8) Change the load of the tested bolt and repeat operations 2-6 for at least 10 times to complete the calibration process of the tested bolt
(9) Correlate the stress and $R_{\mathrm{LC}-\mathrm{L}}$ to get the calibration data. Then, nonlinear fitting is conducted to estimate the value of $a, b$, and $c$ in equation (30) to get the evaluation model of axial stress of the bolt

(10) Evaluate the axial stress by the nonlinear evaluation model

\section{Experiment}

4.1. Setup. The JSR PRC50 ultrasonic transceiving card with gain range from $-14 \mathrm{~dB}$ to $60 \mathrm{~dB}$ is selected as the actuator for the ultrasonic transducers. And the AD-link PCIe-9852 oscilloscope with sampling rates upper to $200 \mathrm{MHz}$ is selected as the data acquisition hardware. They are integrated into a portable industrial computer, as shown in Figure 5. Then, the ultrasonic calibration/measurement software to implement excitation, acquisition, and processing of ultrasonic signals is programmed based on LABVIEW. The Dakota PT7 magnetic longitudinal ultrasonic transducer is chosen as the L-wave source and an Olympus V-156RM transverse ultrasonic transducer is chosen as the S-wave source. The central frequency of both probes is $5 \mathrm{MHz}$. Two types of bolts with different specifications and materials are selected as the test samples. The specification of the specimens is listed in Table 3. The CTM2200S tensile testing machine is utilized to simulate the loading condition with the maximum load $2000 \mathrm{~kg}$ and load accuracy $\pm 0.1 \%$.

4.2. TOF Estimation. Figure 6 shows the ultrasonic signal collected in sample M8 $\times 104$ excited by the PT7 L-wave transducer. According to equations (3) and (4), the waveforms of LLL and LC can be recognized obviously, as shown in Figure 6. And the TOF of LLL can almost be obtained directly because its waveform is clearly a single sharp pulse. While the waveform of LC is consisted of several peaks with similar amplitudes and different phases, its TOF cannot be directly determined. Figure 7 shows the waveform of LC intercepted from the original signal and its Gabor expansion. From Figure 7(b), it can be observed that there is a sharp peak in the diagram of Gabor expansion, and the TOF of the intercepted waveform can be obtained by the proposed particle filter method.

In order to eliminate the influence of temperature on the sound velocity, the whole experiment is carried out under constant temperature $\left(20^{\circ} \mathrm{C}\right)$. The loading range is 0 $190 \mathrm{MPa}$ and the loading step is $10 \mathrm{MPa}$. The elongation of TOF caused by the acoustoelastic effect is around 1 nanosecond per MPa, but the minimum sampling period of PCIe9852 is $5 \mathrm{~ns}$. There is a certain probability that the change of the signal cannot be collected intact, so the onedimensional fast Fourier interpolation method is introduced to enhance the sample rate. After being processed by tentime upsampling, the time resolution of the system is upgraded to $0.5 \mathrm{~ns}$. The estimated TOFs under different stress status using the proposed method are shown in Tables 4 and 5, in which $R_{\mathrm{L}-\mathrm{S}}$ is the time ratio of longitudinal wave (L) to shear wave (S) and $R_{\mathrm{LC}-\mathrm{L}}$ is the time ratio of converted wave (LC) to longitudinal wave. 


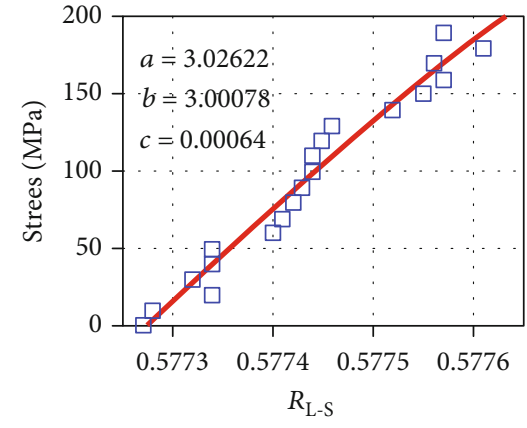

(a) Sample M8 $\times 104$ result of the L-S method

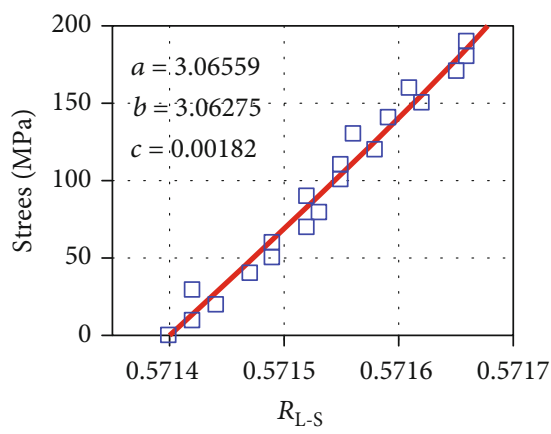

(c) Sample M12 $\times 200$ result of the L-S method

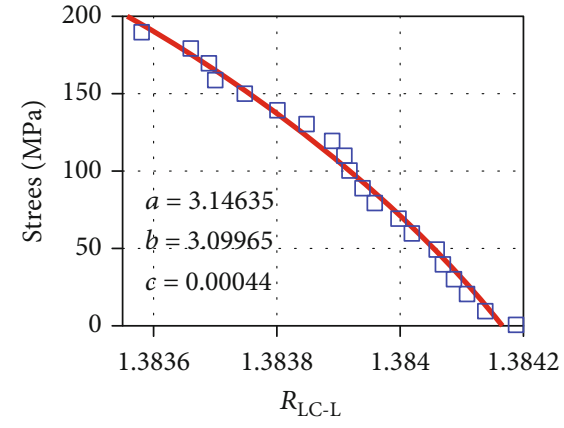

(b) Sample M8 $\times 104$ result of the LC-L method

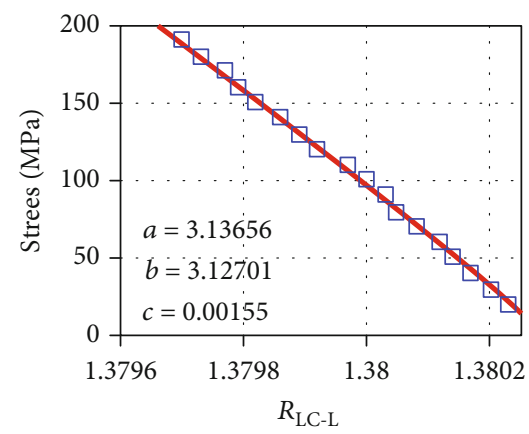

(d) Sample M12 $\times 200$ result of the LC-L method

Figure 8: Calibration results.

TABLE 6: Measurement result of sample M8 × 104 .

\begin{tabular}{lcccccc}
\hline $\begin{array}{l}\text { Preset } \\
\begin{array}{l}\text { stress } \\
(\mathrm{MPa})\end{array}\end{array}$ & $R_{\mathrm{LC}-\mathrm{L}}$ & $R_{\mathrm{L}-\mathrm{S}}$ & $\begin{array}{c}\text { Result of } \\
\text { LC-L } \\
(\mathrm{MPa})\end{array}$ & $\begin{array}{c}\text { Error } \\
\text { of LC- } \\
\mathrm{L}\end{array}$ & $\begin{array}{c}\text { Result } \\
\text { of L-S } \\
(\mathrm{MPa})\end{array}$ & $\begin{array}{c}\text { Error } \\
\text { of L-S }\end{array}$ \\
\hline 20 & 1.38410 & 0.57731 & 21.012 & $5.06 \%$ & 23.531 & $17.65 \%$ \\
40 & 1.38407 & 0.57733 & 38.975 & $-2.56 \%$ & 41.891 & $4.72 \%$ \\
60 & 1.38403 & 0.57741 & 61.211 & $2.02 \%$ & 62.301 & $3.83 \%$ \\
80 & 1.38397 & 0.57742 & 82.007 & $2.50 \%$ & 82.990 & $3.73 \%$ \\
100 & 1.38394 & 0.57744 & 101.250 & $1.25 \%$ & 95.699 & $-4.30 \%$ \\
120 & 1.38388 & 0.57748 & 118.271 & $-1.43 \%$ & 122.256 & $1.88 \%$ \\
140 & 1.38379 & 0.57751 & 142.571 & $0.11 \%$ & 134.368 & $-3.88 \%$ \\
160 & 1.38372 & 0.57757 & 163.254 & $2.03 \%$ & 163.021 & $1.88 \%$ \\
180 & 1.38363 & 0.57760 & 180.201 & $0.11 \%$ & 181.336 & $0.74 \%$ \\
200 & 1.38357 & 0.57763 & 198.367 & $-0.82 \%$ & 208.214 & $4.10 \%$ \\
\hline
\end{tabular}

4.3. Calibration/Measurement. The axial stress calibration tests of bolts are carried out using the L-S method and the LC-L method, respectively. Figure 8 shows the calibration curves obtained from nonlinear fitting by Levenberg-Marquardt method. It can be clearly observed that the results of the LC-L method are significantly better than the results of the L-S method from Figure 8. The correlation coefficients between the fitting model and original data of the LC-L method are 0.9957 for sample M8 $\times 104$ and 0.9994 for sample M12 $\times$ 200. The correlation coefficients of the L-S method are 0.9487 for sample M8 × 104 and 0.94664 for sample M12 × 200 .

Another two bolts exactly the same as the samples are chosen to test the validity of the proposed method. After
TABle 7: Measurement result of sample M12 × 200 .

\begin{tabular}{lcccccc}
\hline $\begin{array}{l}\text { Preset } \\
\text { stress } \\
(\mathrm{MPa})\end{array}$ & $R_{\mathrm{LC}-\mathrm{L}}$ & $R_{\mathrm{L}-\mathrm{S}}$ & $\begin{array}{c}\text { Result of } \\
\text { LC-L } \\
(\mathrm{MPa})\end{array}$ & $\begin{array}{c}\text { Error } \\
\text { of LC- } \\
\mathrm{L}\end{array}$ & $\begin{array}{c}\text { Result } \\
\text { of L-S } \\
(\mathrm{MPa})\end{array}$ & $\begin{array}{c}\text { Error of } \\
\text { L-S }\end{array}$ \\
\hline 20 & 1.38022 & 0.57145 & 22.103 & $10.51 \%$ & 17.655 & $-11.72 \%$ \\
40 & 1.38017 & 0.57147 & 38.127 & $-4.68 \%$ & 40.239 & $0.59 \%$ \\
60 & 1.38013 & 0.57148 & 58.699 & $-2.16 \%$ & 55.627 & $-7.28 \%$ \\
80 & 1.38004 & 0.57154 & 81.475 & $1.84 \%$ & 88.044 & $10.05 \%$ \\
100 & 1.38001 & 0.57157 & 96.583 & $-3.42 \%$ & 105.215 & $5.21 \%$ \\
120 & 1.37992 & 0.57159 & 119.338 & $-0.55 \%$ & 123.144 & $2.62 \%$ \\
140 & 1.37985 & 0.57161 & 140.152 & $-0.10 \%$ & 144.878 & $3.48 \%$ \\
160 & 1.37979 & 0.57162 & 163.254 & $2.03 \%$ & 157.716 & $-1.42 \%$ \\
180 & 1.37974 & 0.57165 & 179.563 & $-0.24 \%$ & 182.771 & $1.53 \%$ \\
200 & 1.37969 & 0.57164 & 199.361 & $-0.32 \%$ & 204.885 & $2.44 \%$ \\
\hline
\end{tabular}

loading by the tensile testing machine at the step of $20 \mathrm{MPa}$, the TOFs under different stress status using the proposed method are recognized. And then, the LevenbergMarquardt models of calibration results are utilized to evaluate the stress of bolts. The evaluation results are shown in Tables 6 and 7 and Figure 9. In Figure 9, the yellow bar represents the absolute error using the proposed method and the blue bar refers to the absolute error of the L-S method. According to the results, the average evaluation errors of the LC-L method and the L-S method for sample M8 $\times 104$ are $1.789 \%$ and $4.671 \%$, respectively. And the average errors of sample $\mathrm{M} 12 \times 200$ are $2.582 \%$ and $4.634 \%$. As a result, the proposed method has a higher 


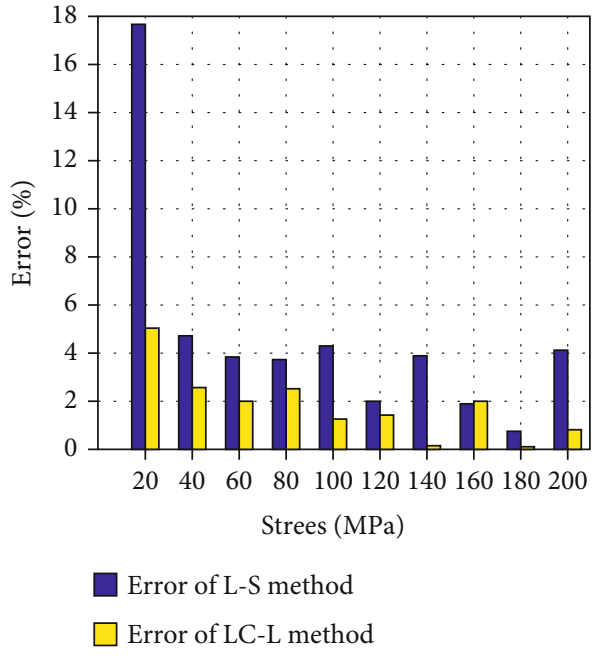

(a) Error of the sample M8 $\times 104$

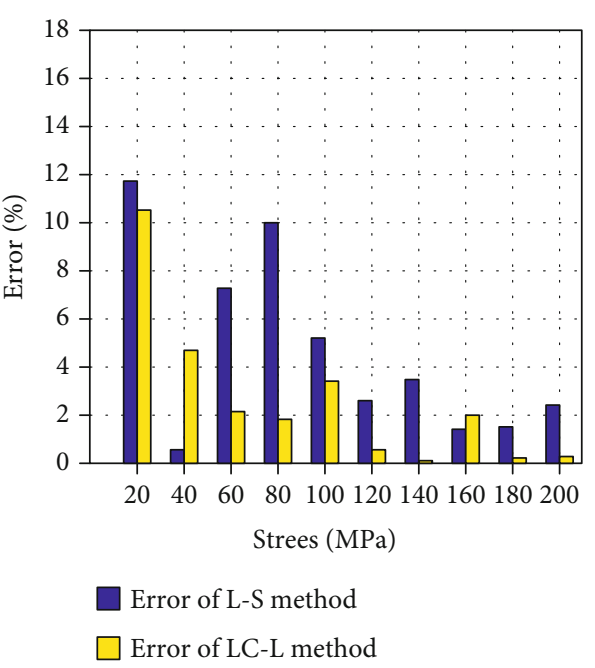

(b) Error of the sample M12 $\times 200$

Figure 9: Measurement error.

accuracy as it utilizes a single longitudinal ultrasound. However, the accuracy of the L-S method is relatively low for the difference of the coupling conditions of $\mathrm{L}$-wave and S-wave transducers.

\section{Conclusions}

In order to determine the parameters of mode-converted wave in single-source ultrasound for bolt stress evaluation, a time-frequency parameter recognition method based on the Gabor transform and a new bolt axial stress evaluation model are proposed in this paper. After analyzing the propagation and the mode conversion process of ultrasonic longitudinal wave in mounted solid, a parameter recognition method based on the Gabor transform to achieve the accurate TOF of the mode-converted wave is introduced to overcome the distortion and aliasing in single-source ultrasonic signal and its effectiveness is proved by simulation. Based on the mode conversion of single-source ultrasound and the acoustoelastic effect, a nonlinear evaluation model of the axial stress is put forward. The proposed method is compared with the commonly used L-S method by a series of calibrations and experiments. And the experiment results show that the proposed method can effectively detect the bolt axial stress ranging from $20 \mathrm{MPa}$ to $200 \mathrm{MPa}$ with a higher accuracy. As a result, the proposed method is more efficient and applicable in detecting the connection status of bolted joints than the traditional L-S method.

\section{Data Availability}

The measurement data used to support the findings of this study are included within the article.

\section{Conflicts of Interest}

The authors declare no conflict of interest.

\section{Acknowledgments}

This research was funded by the National Natural Science Foundation of China (grant number 51374264) and the Science and Technology Major Project of Chongqing (grant number cstc2018jszx-cyztzxX0032).

\section{References}

[1] L. U. Peng, Y. U. Le, L. Hua et al., "Research on screw bolt's pre-tightening force by coating photoelasticity," Chinese quarterly of mechanics, vol. 2, pp. 275-278, 2013.

[2] X. Wang, Q. Sun, F. Zhang, J. Duan, H. Liang, and H. Wang, "Design on static resistance strain gauge of mine roof bolt based on ATMega16L," Journal of Liaoning Technical University (Natural Science), vol. 2, pp. 288-292, 2011.

[3] Z. G. Guo and Z. Sun, "Piezoelectric impedance based prestress force monitoring for PSC beam," Advanced Materials Research, vol. 255-260, pp. 742-746, 2011.

[4] J. Xu, C. Wang, H. Li, C. Zhang, J. Hao, and S. Fan, "Health monitoring of bolted spherical joint connection based on active sensing technique using piezoceramic transducers," Sensors, vol. 18, no. 6, p. 1727, 2018.

[5] F. Wang, Y. J. Xu, L. Wu, L. Li, D. Liu, and L. Zhu, "A PEFKSand CP-ABE-based distributed security scheme in interestcentric opportunistic networks," International Journal of Distributed Sensor Networks, vol. 2013, 10 pages, 2013.

[6] S. G. Joshi and R. G. Pathare, "Ultrasonic instrument for measuring bolt stress," Ultrasonics, vol. 22, no. 6, pp. 261-269, 1984.

[7] J. S. Heyman and E. Churn, "Ultrasonic measurement of axial stress," Journal of Testing and Evaluation, vol. 10, no. 5, pp. 202-211, 1992.

[8] D. I. Crecraft, "The measurement of applied and residual stresses in metals using ultrasonic waves," Journal of Sound and Vibration, vol. 5, no. 1, pp. 173-192, 1967.

[9] N. Y. Nikitina and L. A. Ostrovsky, "An ultrasonic method for measuring stresses in engineering materials," Ultrasonics, vol. 35, no. 8, pp. 605-610, 1998. 
[10] A. C. Holt, B. Cunningham, G. C. Johnson, and D. Auslander, "An ultrasonic technique for axial bolt-stress determination," Review of Progress in Quantitative Nondestructive Evaluation, pp. 1549-1557, 1987.

[11] D. E. Bray, "Application of ultrasonic stress measurement to engineering components," Journal of the Acoustical Society of America, vol. 102, no. 5, pp. 3082-3082, 1997.

[12] H. U. Youbin, X. U. Yong, and Z. Jianmin, “Application of ultrasonic measurement technique for bolt stress to tightening of mill shell," Mining \& Processing Equipment, vol. 8, pp. 112116, 2012.

[13] H. Yasui, H. Tanaka, I. Fujii, and K. Kawashima, "Ultrasonic measurement of axial stress in short Bolts with consideration of nonlinear deformation," JSME International Journal Series A Solid Mechanics and Material Engineering, vol. 42, no. 1, pp. 111-118, 1999.

[14] N. Kim and M. Hong, "Measurement of axial stress using mode-converted ultrasound," NDT \& E International, vol. 42, no. 3, pp. 164-169, 2009.

[15] R. Demirli and J. Saniie, "Model-based estimation of ultrasonic echoes part I: analysis and algorithms," IEEE Transaction Ultrasonic, Ferro Electrics and Frequency Control, vol. 48, no. 3, pp. 787-802, 2001.

[16] L. Angrisani, A. Baccigalupi, and R. SchianoLoMoriello, "A measurement method based on Kalman filtering for ultrasonic time-of-flight estimation," IEEE Transactions on Instrumentation and Measurement, vol. 55, no. 2, pp. 442-448, 2006.

[17] Z. K. Lu, C. Yang, and J. W. Wang, "Gabor transform based time-frequency estimation of ultrasonic echo signal," Journal of Electronics \& Information Technology, vol. 3, pp. 652-657, 2013.

[18] D. W. Wang, Z. B. Wang, Y. X. Chen et al., "Ultrasonic echo processing method based on dual-Gaussian attenuation model," Acta Physica Sinica, vol. 8, pp. 168-176, 2019.

[19] H. Huang, W. Li, D. A. Luo, D. W. Qiu, and Y. Gao, “An improved particle filter algorithm for geomagnetic indoor positioning," Journal of Sensors, vol. 2018, 9 pages, 2018.

[20] M. Mishra, J. Odelius, A. Thaduri, A. Nissen, and M. Rantatalo, "Particle filter-based prognostic approach for railway track geometry," Mechanical systems and signal processing, vol. 96, pp. 226-238, 2017.

[21] K. Li and L. Chang, "Robust Gaussian particle filter based on modified likelihood function," IET Science, Measurement \& Technology, vol. 12, no. 1, pp. 132-137, 2018.

[22] Y. Kim, W. Chung, and D. Hong, "Indoor parking localization based on dual weighted particle filter," International Journal of Precision Engineering \& Manufacturing, vol. 19, no. 2, pp. 293-298, 2018.

[23] J. L. Rose and P. B. Nagy, "Ultrasonic waves in solid media," Journal of the Acoustical Society of America, vol. 107, no. 4, pp. 1807-1808, 2000. 